\title{
Reducing Drug Wastage at Ward Level: Using Failure Mode Effects Analysis (Fmea) Tool
}

\author{
${ }^{1}$ AR.Abdul Aziz, ${ }^{2}$ M B.Nishazini, ${ }^{3}$ H. Fareza, ${ }^{4}$ N A. Azizan \\ ${ }^{1,2,3}$ KPJ Seremban Specialist Hospital \\ ${ }^{4}$ University Malaysia Pahang
}

\begin{abstract}
For year 2010, total value of drugs disposed was RM 8,575.50 due to expired or spoiled items returned from wards. The main reason was due to the failure of nurses to check the stock regularly. They only do the checking upon ordering of new stock from Pharmacy department. FMEA tool was selected by the Management to solve this problem. A special committee was set up to implement the FMEA in January 2011.The committee thru brainstorming session had developed seven steps in implementing the FMEA. At step three, Risk Priority Number (RPN) was determined. At step four with the highest RPN of 36, it was found that the failure mode of the system were due to failure of checking stock at ward level, not double checking of the drug received and wrong storage of drug at ward level. A new supply system was implemented at step five and monitoring of drug disposed started in July 2011. In this system, pharmacy staff will go to the wards once a month to check stock of drugs kept at ward level. After the implementation of the new process, RPN was calculated again and it was found that the value was between 3 to 8 which was considered as low. Monitoring of compliance was done using ward check form based on storage condition, par level, labeling and packaging and non- conformances for spoilt and expired items. Compliance in term of drug storage was $100 \%$ and labeling compliance was $97.2 \%$. However the compliance on par level was only $81.9 \%$ and in term of expiry date validity, the compliance was $96.5 \%$. Based on FMEA, it was found that the main reason for expired/spoiled drugs is because of insufficient checking of ward stocks. Corrective actions are ongoing to improve further the process of supply by re-designing a new form of ward checking, re-modifying indenting process of ward stocks via the HITS system and conducting training and awareness. After implementing the new system, the value of drug disposed was RM 3,060.78 which was 94\% lower. Therefore the study has shown remarkable results in reducing the amount of drug disposal which will help the organization to reduce the risk to patients and avoid wastage.
\end{abstract}

Key words: Drug wastage, Failure Mode Effect Analysis(FMEA), Risk Priority Number (RPN)

\section{Introduction}

KPJ Seremban Specialist Hospital is the subsidiary of KPJ Healthcare Berhad, the biggest chain of private hospital in Malaysia .Pharmacy department received high return of short expiry, expired or spoiled drugs. For year 2010, total value of drug disposed was RM 8,575.50 due to expired or spoiled items returned from wards. Therefore patients are at risk of getting expired or spoiled drugs during their admission in the hospital. Based on the analysis it was found that that checking of drugs was only done by the nurses when they plan to indent new stock from Pharmacy. Some drugs are slow moving and remain in stock until used. As a result those drugs may have expired without anyone noticing. In order to overcome the problem, FMEA was implemented to look at the ward stock indent processes to identify the failure mode, its effects and causes and subsequently designing a new plan to minimize the risk and losses. The ultimate goal of FMEA is to prevent bad outcomes and prevent harm. The greatest strength of FMEA lies in its ability to focus users on the process of redesigning potential problematic processes to prevent the occurrence of failures (JCI,FMEA )

\section{Objectives}

1.To find out the value of drugs disposed from ward's level

2.To identify the failure mode of the system of supply

3.To implement a new system to reduce the failure rate

4.To monitor the performance of the new system

\section{Methodology}

In January 2011, the management decided to implement FMEA in pharmacy department based on the value of drugs disposal from January to December 2010. The implementation of FMEA will follow the following steps 


\section{STEP ONE:}

To form a committee of the FMEA at the hospital level. The committee was headed by the Chief Pharmacist assisted by three nursing personals, three pharmacy staff and Quality Executive .

FMEA is a commonly used prospective risk assessment approach in health care. It is time consuming and resource intensive and team performance

is crucial for FMEA success (Wetterneck, 2009)

\section{STEP TWO:}

To assess the current ward stock indenting process. The assessment was done using FMEA worksheet (McDermott, 2009)

\section{WARD STOCK INDENTING PROCESS}

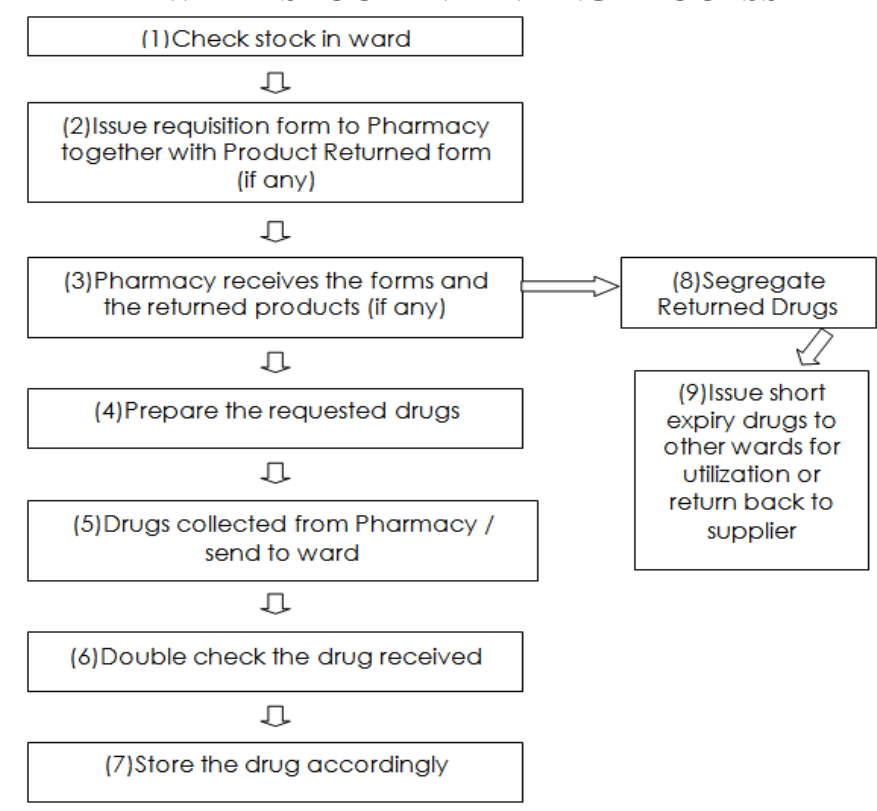

Nurses checked their stock when they plan to indent drugs from Pharmacy. During checking, nurses are required to check par level, storage, expiry date and physical condition of drugs.

\section{STEP THREE:}

To brainstorming.

The process of brainstorming follows the techniques of Tischler, 2010. During brainstorming activity, potential failure modes, its effects and causes within each process are tabulated. Scoring the FMEA is done according to the Guidelines below.

\section{SCORING GUIDELINES:}

Key for Severity Rating: Determine the severity of the effect on the patient / organization

\begin{tabular}{|l|l|}
\hline Severity Score & Description \\
\hline 1 & Minor patient / company outcome: No injury, increased LOS or level of care; no incur loss \\
\hline 2 & Moderate patient / company outcome: Injury, Increased LOS or level of care; incurred loss \\
\hline 3 & $\begin{array}{l}\text { Major patient / company outcome: Major medical intervention required, increased LOS and/or } \\
\text { level of care for >3patients; major incurred losses }\end{array}$ \\
\hline 4 & $\begin{array}{l}\text { Catastrophic patient / company outcome: Death or major permanent loss of function, wrong } \\
\text { procedure on wrong patient, medication error / ADR incidents; lawsuits }\end{array}$ \\
\hline
\end{tabular}

Key for Occurence Rating: Determine the frequency of causes occurence

\begin{tabular}{|l|l|}
\hline Occurence Score & Description \\
\hline 1 & Remote: unlikely to occur (sometime in 3-10 years) \\
\hline 2 & Uncommon: Possible to occur (few times in a year) \\
\hline 3 & Occasional: Probably will occur (eg several times in a month) \\
\hline 4 & Frequent: Likely to occur immediately or within a short period \\
\hline
\end{tabular}


Key for Detectability Score: Determine the detectability of problem before affecting patient / organization

\begin{tabular}{|l|l|}
\hline Detectability Score & Description \\
\hline 1 & Easily detectable \\
\hline 2 & Often detectable \\
\hline 3 & Unlikely detected \\
\hline 4 & Rarely detected \\
\hline
\end{tabular}

*Scoring method adapted from: National Centre for Patient Safety, Healthcare Failure Mode and Effect Analysis (HFMEA) Institute for Safe Medication Practices (ISMP) 2005

Risk Priority Number (RPN): Determines where the greatest hazard lies. It is a measure for comparison within one process only; it is not a measure for comparing risk between processes or organizations (Institute of Health Improvement)

RPN $=$ Severity $x$ Occurrence $x$ Detectability

IV. Failure Modes And Effects Analysis

\begin{tabular}{|c|c|c|c|c|c|c|c|c|c|c|}
\hline $\begin{array}{l}\mathbf{N} \\
\mathbf{0}\end{array}$ & Process & $\begin{array}{l}\text { Failure } \\
\text { Modes }\end{array}$ & Effects & $\begin{array}{l}\text { Sev } \\
\text { e- } \\
\text { rity }\end{array}$ & $\begin{array}{l}\text { Causes of } \\
\text { Failure }\end{array}$ & $\begin{array}{l}\text { Occ } \\
\text { ur- } \\
\text { renc } \\
\text { es }\end{array}$ & $\begin{array}{l}\text { Dete } \\
\text { ct- } \\
\text { abili } \\
\text { ty }\end{array}$ & $\begin{array}{l}\mathbf{R} \\
\mathbf{P} \\
\mathbf{N}\end{array}$ & $\begin{array}{l}\text { Ra } \\
\text { nk }\end{array}$ & $\begin{array}{lr}\text { Action } & \text { to } \\
\text { Reduce } & \text { Failure } \\
\text { Mode } & \end{array}$ \\
\hline \multirow[t]{8}{*}{1} & \multirow[t]{8}{*}{$\begin{array}{l}\text { Check } \\
\text { stock } \\
\text { level in } \\
\text { ward }\end{array}$} & \multirow[t]{4}{*}{$\begin{array}{l}\text { SN did not } \\
\text { check ward } \\
\text { stock level. }\end{array}$} & \multirow[t]{2}{*}{$\begin{array}{l}\text { No } \\
\text { replenishment, } \\
\text { stock not } \\
\text { sufficient }\end{array}$} & 2 & $\begin{array}{l}\text { Inadequat } \\
\text { e staffing, }\end{array}$ & 3 & 3 & $\begin{array}{l}1 \\
8\end{array}$ & 3 & $\begin{array}{l}\text { Reschedule } \\
\text { staffing pattern }\end{array}$ \\
\hline & & & & 2 & $\begin{array}{l}\text { Lack of } \\
\text { making } \\
\text { the check } \\
\text { a priority }\end{array}$ & 3 & 3 & $\begin{array}{l}1 \\
8\end{array}$ & 3 & $\begin{array}{l}\text { Engaging staff in } \\
\text { culture of safety } \\
\text { - importance of } \\
\text { daily checking }\end{array}$ \\
\hline & & & \multirow{2}{*}{$\begin{array}{l}\text { Patient may } \\
\text { not get } \\
\text { medication on } \\
\text { time during } \\
\text { emergency }\end{array}$} & 4 & $\begin{array}{l}\text { Replenish } \\
\text { ment } \\
\text { failure }\end{array}$ & 3 & 3 & $\begin{array}{l}3 \\
6\end{array}$ & 1 & $\begin{array}{lr}\text { Integrate } & \text { check } \\
\text { process } & \text { into } \\
\text { daily task } & \end{array}$ \\
\hline & & & & 4 & $\begin{array}{l}\text { Staff } \\
\text { unaware } \\
\text { of stock } \\
\text { level }\end{array}$ & 2 & 3 & $\begin{array}{l}2 \\
4\end{array}$ & 2 & $\begin{array}{l}\text { Daily monitoring } \\
\text { of stock level - } \\
\text { bin card }\end{array}$ \\
\hline & & $\begin{array}{l}\text { Check } \\
\text { inadequate }\end{array}$ & $\begin{array}{l}\text { Replenishment } \\
\text { not according } \\
\text { to usage }\end{array}$ & 1 & $\begin{array}{l}\text { Inefficient } \\
\text { workflow }\end{array}$ & 4 & 4 & $\begin{array}{l}1 \\
6\end{array}$ & 4 & $\begin{array}{l}\text { Daily monitoring } \\
\text { of stock level - } \\
\text { bin card to check } \\
\text { on usage }\end{array}$ \\
\hline & & \multirow{3}{*}{$\begin{array}{l}\text { Staff not } \\
\text { sure how to } \\
\text { check and } \\
\text { indent }\end{array}$} & \multirow[t]{2}{*}{$\begin{array}{l}\text { Wrongly } \\
\text { indent item }\end{array}$} & 2 & New staff & 2 & 3 & $\begin{array}{l}1 \\
2\end{array}$ & 5 & Training \\
\hline & & & & 2 & $\begin{array}{l}\text { Staff } \\
\text { competenc } \\
\text { y }\end{array}$ & 2 & 3 & $\begin{array}{l}1 \\
2\end{array}$ & 5 & $\begin{array}{l}\text { Training and } \\
\text { supervision }\end{array}$ \\
\hline & & & $\begin{array}{l}\text { Over stock / } \\
\text { Understock }\end{array}$ & 2 & $\begin{array}{l}\text { Not aware } \\
\text { on drug } \\
\text { usage }\end{array}$ & 3 & 3 & $\begin{array}{l}1 \\
8\end{array}$ & 3 & Drug par level \\
\hline $\begin{array}{l}\mathbf{N} \\
\mathbf{0}\end{array}$ & Process & $\begin{array}{l}\text { Failure } \\
\text { Modes }\end{array}$ & Effects & $\begin{array}{l}\text { Sev } \\
\text { e- } \\
\text { rity }\end{array}$ & $\begin{array}{l}\text { Causes of } \\
\text { Failure }\end{array}$ & $\begin{array}{l}\text { Occ } \\
\text { ur- } \\
\text { renc } \\
\text { es }\end{array}$ & $\begin{array}{l}\text { Dete } \\
\text { ct- } \\
\text { abili } \\
\text { ty }\end{array}$ & $\begin{array}{l}\mathbf{R} \\
\mathbf{P} \\
\mathbf{N}\end{array}$ & $\begin{array}{l}\text { Ra } \\
\text { nk }\end{array}$ & $\begin{array}{lr}\text { Action } & \text { to } \\
\text { Reduce } & \text { Failure } \\
\text { Mode } & \end{array}$ \\
\hline \multirow[t]{2}{*}{2} & \multirow[t]{2}{*}{$\begin{array}{l}\text { Issue } \\
\text { requisitio } \\
\mathrm{n} \text { form to } \\
\text { Pharmacy } \\
\text { together } \\
\text { with } \\
\text { Product } \\
\text { Returned } \\
\text { form (if }\end{array}$} & \multirow[t]{2}{*}{$\begin{array}{l}\text { Wrong item } \\
\text { entered }- \\
\text { Order not } \\
\text { complete }\end{array}$} & \multirow[t]{2}{*}{$\begin{array}{l}\text { Supply wrong } \\
\text { medication }\end{array}$} & 4 & $\begin{array}{l}\text { Illegible } \\
\text { hand } \\
\text { writing }- \\
\text { staff } \\
\text { misread } \\
\text { drug } \\
\text { indent }\end{array}$ & 3 & 2 & $\begin{array}{l}2 \\
4\end{array}$ & 2 & $\begin{array}{l}\text { Printed order } \\
\text { computerised }\end{array}$ \\
\hline & & & & 4 & LASA & 3 & 2 & 2 & 2 & Write \\
\hline
\end{tabular}




\begin{tabular}{|c|c|c|c|c|c|c|c|c|c|c|}
\hline & \multirow[t]{6}{*}{ any) } & & & & drug & & & 4 & & CAPITAL letter \\
\hline & & $\begin{array}{l}\text { Forms not } \\
\text { received by } \\
\text { Pharmacy }\end{array}$ & $\begin{array}{lr}\text { No } & \text { supply } \\
\text { made } & \text { or } \\
\text { returned drugs } \\
\text { received }\end{array}$ & 2 & $\begin{array}{l}\text { Forms } \\
\text { misplace / } \\
\text { missing }\end{array}$ & 2 & 2 & 8 & 6 & $\begin{array}{l}\text { Form should be } \\
\text { accompanied } \\
\text { with trolley/ } \\
\text { medication box }\end{array}$ \\
\hline & & \multirow{4}{*}{$\begin{array}{l}\text { Staff not } \\
\text { sure how to } \\
\text { check and } \\
\text { indent }\end{array}$} & \multirow[t]{2}{*}{$\begin{array}{l}\text { Wrongly } \\
\text { indent item }\end{array}$} & 4 & New staff & 2 & 3 & $\begin{array}{l}2 \\
4\end{array}$ & 2 & Training \\
\hline & & & & 4 & $\begin{array}{l}\text { Staff } \\
\text { competenc } \\
\mathrm{y}\end{array}$ & 2 & 3 & $\begin{array}{l}2 \\
4\end{array}$ & 2 & $\begin{array}{l}\text { Training and } \\
\text { supervision }\end{array}$ \\
\hline & & & $\begin{array}{l}\text { Over stock / } \\
\text { Understock }\end{array}$ & 2 & $\begin{array}{l}\text { Not aware } \\
\text { on drug } \\
\text { usage }\end{array}$ & 3 & 3 & $\begin{array}{l}1 \\
8\end{array}$ & 3 & Drug par level \\
\hline & & & $\begin{array}{l}\text { Short expiry / } \\
\text { expired drugs } \\
\text { not returned to } \\
\text { Pharmacy }\end{array}$ & 4 & $\begin{array}{l}\text { No regular } \\
\text { checking }\end{array}$ & 2 & 3 & $\begin{array}{l}2 \\
4\end{array}$ & 2 & $\begin{array}{l}\text { Short expiry } \\
\text { drugs to be tag } \\
\text { with reminder } \\
\text { label }\end{array}$ \\
\hline $\begin{array}{l}\mathbf{N} \\
\mathbf{0}\end{array}$ & Process & $\begin{array}{l}\text { Failure } \\
\text { Modes }\end{array}$ & Effects & $\begin{array}{l}\text { Sev } \\
\text { e- } \\
\text { rity }\end{array}$ & $\begin{array}{l}\text { Causes of } \\
\text { Failure }\end{array}$ & $\begin{array}{l}\text { Occ } \\
\text { ur- } \\
\text { renc } \\
\text { es }\end{array}$ & $\begin{array}{l}\text { Dete } \\
\text { ct- } \\
\text { abili } \\
\text { ty } \\
\end{array}$ & $\begin{array}{l}\mathbf{R} \\
\mathbf{P} \\
\mathbf{N}\end{array}$ & $\begin{array}{l}\text { Ra } \\
\text { nk }\end{array}$ & $\begin{array}{lr}\text { Action } & \text { to } \\
\text { Reduce } & \text { Failure } \\
\text { Mode } & \end{array}$ \\
\hline \multirow[t]{3}{*}{3} & \multirow[t]{3}{*}{$\begin{array}{l}\text { Pharmacy } \\
\text { receives } \\
\text { the forms } \\
\text { and the } \\
\text { returned } \\
\text { products } \\
\text { (if any) }\end{array}$} & \multirow[t]{3}{*}{$\begin{array}{l}\text { Delay in } \\
\text { receiving } \\
\text { form or not } \\
\text { received at } \\
\text { all }\end{array}$} & \multirow[t]{2}{*}{$\begin{array}{l}\text { No supply - } \\
\text { insufficient } \\
\text { stock }\end{array}$} & 2 & $\begin{array}{l}\text { Misplace } \\
\text { request } \\
\text { form }\end{array}$ & 2 & 1 & 4 & 8 & $\begin{array}{l}\text { System for top } \\
\text { up to be } \\
\text { designed } \\
\text { include } \\
\text { scheduling for } \\
\text { topping up. } \\
\text { Indent form } \\
\text { should be } \\
\text { accompanied } \\
\text { with trolley/ } \\
\text { medication box }\end{array}$ \\
\hline & & & & 2 & $\begin{array}{l}\text { Inefficient } \\
\text { process } \\
\text { for } \\
\text { sending } \\
\text { orders }\end{array}$ & 2 & 1 & 4 & 8 & $\begin{array}{l}\text { Work on } \\
\text { workflow for top } \\
\text { up system } \\
\text { Training to all } \\
\text { staff involved }\end{array}$ \\
\hline & & & $\begin{array}{l}\text { Short expiry / } \\
\text { expired drugs } \\
\text { not returned }\end{array}$ & 4 & $\begin{array}{l}\text { Misplace } \\
\text { request } \\
\text { form }\end{array}$ & 2 & 1 & 4 & 8 & $\begin{array}{l}\text { Returned drug } \\
\text { should be } \\
\text { accompanied } \\
\text { with trolley / } \\
\text { medication box }\end{array}$ \\
\hline \multirow[t]{3}{*}{4} & \multirow[t]{3}{*}{$\begin{array}{l}\text { Prepare } \\
\text { the } \\
\text { requested } \\
\text { drugs }\end{array}$} & $\begin{array}{l}\text { No stock } \\
\text { issue }\end{array}$ & $\begin{array}{l}\text { No supply to } \\
\text { ward } \\
\text { inadequate }\end{array}$ & 2 & $\begin{array}{l}\text { Supplier } \\
\text { out of } \\
\text { stock }\end{array}$ & 2 & 1 & 4 & 8 & $\begin{array}{l}\text { Find alternative } \\
\text { drug to cover } \\
\text { usage } \\
\text { Sms drop/ memo } \\
\text { circulation to all } \\
\text { involved on } \\
\text { substitution } \\
\text { available }\end{array}$ \\
\hline & & \multirow[t]{2}{*}{$\begin{array}{l}\text { Inaccurate } \\
\text { labeling i.e } \\
\text { unclear } \\
\text { printing, } \\
\text { label lost }\end{array}$} & $\begin{array}{l}\text { Wrong drug / } \\
\text { strength } \\
\text { supplied }\end{array}$ & 2 & $\begin{array}{l}\text { Human } \\
\text { errors, } \\
\text { ambiguou } \\
\mathrm{s} \\
\text { informatio } \\
\mathrm{n}\end{array}$ & 1 & 1 & 2 & 9 & $\begin{array}{l}\text { Double checking } \\
\text { procedure } \\
\text { Separation and } \\
\text { alert label for } \\
\text { LASA drug }\end{array}$ \\
\hline & & & $\begin{array}{l}\text { Ineffective } \\
\text { drug - expiry } \\
\text { date unclear }\end{array}$ & 4 & $\begin{array}{l}\text { Equipmen } \\
\mathrm{t} \\
\text { malfunctio } \\
\mathrm{n}\end{array}$ & 1 & 1 & 4 & 8 & $\begin{array}{l}\text { Routine } \\
\text { equipment } \\
\text { maintenance } \\
\text { check }\end{array}$ \\
\hline $\begin{array}{l}\mathbf{N} \\
\mathbf{0}\end{array}$ & Process & $\begin{array}{l}\text { Failure } \\
\text { Modes }\end{array}$ & Effects & $\begin{array}{l}\text { Sev } \\
\text { e- } \\
\text { rity }\end{array}$ & $\begin{array}{l}\text { Causes of } \\
\text { Failure }\end{array}$ & $\begin{array}{l}\text { Occ } \\
\text { ur- } \\
\text { renc } \\
\text { es }\end{array}$ & $\begin{array}{l}\text { Dete } \\
\text { ct- } \\
\text { abili } \\
\text { ty }\end{array}$ & $\begin{array}{l}\mathbf{R} \\
\mathbf{P} \\
\mathbf{N}\end{array}$ & $\begin{array}{l}\text { Ra } \\
\text { nk }\end{array}$ & $\begin{array}{lr}\text { Action } & \text { to } \\
\text { Reduce } & \text { Failure } \\
\text { Mode } & \end{array}$ \\
\hline
\end{tabular}




\begin{tabular}{|c|c|c|c|c|c|c|c|c|c|c|}
\hline \multirow[t]{4}{*}{4} & \multirow[t]{4}{*}{$\begin{array}{l}\text { Prepare } \\
\text { the } \\
\text { requested } \\
\text { drugs } \\
\text { (con't) }\end{array}$} & $\begin{array}{l}\text { Wrong } \\
\text { quantity } \\
\text { supplied }\end{array}$ & $\begin{array}{lr}\text { Stock } & \text { not } \\
\text { sufficient } & \text { to } \\
\text { usage / } & \text { over } \\
\text { supplied } & \end{array}$ & 2 & $\begin{array}{l}\text { Counting } \\
\text { error }\end{array}$ & 2 & 3 & $\begin{array}{l}1 \\
2\end{array}$ & 5 & $\begin{array}{ll}\text { Double } & \text { checking } \\
\text { on } & \text { receiving } \\
\text { drug } & \end{array}$ \\
\hline & & $\begin{array}{l}\text { Supplied } \\
\text { short expiry } \\
\text { / expired } \\
\text { drug }\end{array}$ & $\begin{array}{l}\text { ADR, allergic } \\
\text { reaction or } \\
\text { ineffective } \\
\text { treatment if } \\
\text { administered } \\
\text { to patient }\end{array}$ & 4 & $\begin{array}{l}\text { Not } \\
\text { practice } \\
\text { FIFO } \\
\text { Lack of } \\
\text { monitorin } \\
\mathrm{g} \text { of stock } \\
\text { expiry }\end{array}$ & 2 & 3 & $\begin{array}{l}2 \\
4\end{array}$ & 2 & $\begin{array}{l}\text { Double checking } \\
\text { on receiving } \\
\text { drug } \\
\text { Routine check } \\
\text { on expiry and } \\
\text { separate drugs } \\
\text { on immediate } \\
\text { use area } \\
\text { FIFO } \\
\text { arrangement }\end{array}$ \\
\hline & & \multirow[t]{2}{*}{$\begin{array}{l}\text { Preparation } \\
\text { of drug } \\
\text { delay due to } \\
\text { various } \\
\text { reasons i.e. } \\
\text { insufficient } \\
\text { bottles, staff } \\
\text { in-charge on } \\
\text { sick leave }\end{array}$} & \multirow[t]{2}{*}{$\begin{array}{l}\text { Supply to } \\
\text { ward } \\
\text { inadequate and } \\
\text { delayed. }\end{array}$} & 2 & $\begin{array}{l}\text { Failure of } \\
\text { dry stock } \\
\text { monitorin } \\
g\end{array}$ & 3 & 1 & 6 & 7 & $\begin{array}{l}\text { Bin card use to } \\
\text { monitor stock } \\
\text { availability of } \\
\text { preparation } \\
\text { supplies. } \\
\text { Appoint staff in- } \\
\text { charge on } \\
\text { monitoring dry } \\
\text { stock } \\
\text { consistently }\end{array}$ \\
\hline & & & & 2 & $\begin{array}{l}\text { Inadequat } \\
\text { e staffing }\end{array}$ & 3 & 1 & 6 & 7 & $\begin{array}{l}\text { Replacement } \\
\text { staff to cover } \\
\text { duty }\end{array}$ \\
\hline $\begin{array}{l}\mathbf{N} \\
\mathbf{0}\end{array}$ & Process & $\begin{array}{l}\text { Failure } \\
\text { Modes }\end{array}$ & Effects & $\begin{array}{l}\text { Sev } \\
\text { e- } \\
\text { rity }\end{array}$ & $\begin{array}{l}\text { Causes of } \\
\text { Failure }\end{array}$ & $\begin{array}{l}\text { Occ } \\
\text { ur- } \\
\text { renc } \\
\text { es }\end{array}$ & $\begin{array}{l}\text { Dete } \\
\text { ct- } \\
\text { abili } \\
\text { ty } \\
\end{array}$ & $\begin{array}{l}\mathbf{R} \\
\mathbf{P} \\
\mathbf{N}\end{array}$ & $\begin{array}{l}\text { Ra } \\
\text { nk }\end{array}$ & $\begin{array}{lr}\text { Action } & \text { to } \\
\text { Reduce } & \text { Failure } \\
\text { Mode } & \end{array}$ \\
\hline \multirow[t]{6}{*}{5} & \multirow[t]{6}{*}{$\begin{array}{l}\text { Drug } \\
\text { collected } \\
\text { from } \\
\text { Pharmacy } \\
\text { / send to } \\
\text { ward }\end{array}$} & \multirow[t]{3}{*}{$\begin{array}{l}\text { Delay in } \\
\text { delivery } \quad / \\
\text { collection }\end{array}$} & \multirow[t]{3}{*}{$\begin{array}{l}\text { Shortage of } \\
\text { stock in ward }\end{array}$} & 2 & $\begin{array}{l}\text { Human } \\
\text { errors i.e. } \\
\text { Staff } \\
\text { forgot to } \\
\text { collect / } \\
\text { send }\end{array}$ & 2 & 2 & 8 & 6 & $\begin{array}{l}\text { Establish top up } \\
\text { system time } \\
\text { frame for } \\
\text { collection } \\
\text { delivery } \\
\text { Assign staff for } \\
\text { delivery } \\
\text { collection }\end{array}$ \\
\hline & & & & 2 & $\begin{array}{l}\text { Heavy } \\
\text { workload } \\
-\quad \text { staff } \\
\text { occupied } \\
\text { with other } \\
\text { task } \\
\end{array}$ & 2 & 2 & 8 & 6 & $\begin{array}{l}\text { Assign staff for } \\
\text { delivery } \\
\text { collection }\end{array}$ \\
\hline & & & & 2 & $\begin{array}{l}\text { Inadequat } \\
\text { e staffing } \\
\text { - staff on } \\
\text { leave }\end{array}$ & 2 & 2 & 8 & 6 & $\begin{array}{l}\text { Replacement } \\
\text { staff to cover } \\
\text { duty }\end{array}$ \\
\hline & & $\begin{array}{l}\text { Delivered to } \\
\text { wrong unit }\end{array}$ & $\begin{array}{l}\text { Overstock / } \\
\text { under stock }\end{array}$ & 2 & $\begin{array}{l}\text { Many } \\
\text { trolleys in } \\
\text { pharmacy } \\
-\quad \text { staff } \\
\text { may get } \\
\text { confused }\end{array}$ & 2 & 1 & 4 & 8 & $\begin{array}{l}\text { Accurately label } \\
\text { each unit's } \\
\text { trolley } \\
\text { Dedicated space } \\
\text { for indent trolley } \\
\text { to avoid } \\
\text { congestion in } \\
\text { Pharmacy }\end{array}$ \\
\hline & & \multirow[t]{2}{*}{$\begin{array}{ll}\text { Drug } & \\
\text { dropped } & - \\
\text { broken } & / \\
\text { missing } & \\
\text { during } \\
\text { transit }\end{array}$} & \multirow[t]{2}{*}{$\begin{array}{ll}\text { Cost } & \text { to } \\
\text { company }- \text { to } \\
\text { replace loss of } \\
\text { drug }\end{array}$} & 2 & $\begin{array}{l}\text { Trolleys } \\
\text { not stable }\end{array}$ & 1 & 2 & 4 & 8 & $\begin{array}{l}\text { Proper } \\
\text { arrangement of } \\
\text { drug on trolley } \\
\text { Routine check } \\
\text { on trolleys }\end{array}$ \\
\hline & & & & 2 & $\begin{array}{l}\text { Security } \\
\text { of drugs } \\
\text { during } \\
\text { transit }\end{array}$ & 2 & 2 & 8 & 6 & $\begin{array}{l}\text { Medication box } \\
\text { use to transfer } \\
\text { drugs }\end{array}$ \\
\hline $\begin{array}{l}\mathbf{N} \\
\mathbf{0}\end{array}$ & Process & $\begin{array}{l}\text { Failure } \\
\text { Modes }\end{array}$ & Effects & $\begin{array}{l}\text { Sev } \\
\text { e- }\end{array}$ & $\begin{array}{l}\text { Causes of } \\
\text { Failure }\end{array}$ & $\begin{array}{l}\text { Occ } \\
\text { ur- }\end{array}$ & $\begin{array}{l}\text { Dete } \\
\text { ct- }\end{array}$ & $\begin{array}{l}\mathbf{R} \\
\mathbf{P}\end{array}$ & $\begin{array}{l}\text { Ra } \\
\text { nk }\end{array}$ & $\begin{array}{lr}\text { Action } & \text { to } \\
\text { Reduce } & \text { Failure }\end{array}$ \\
\hline
\end{tabular}




\begin{tabular}{|c|c|c|c|c|c|c|c|c|c|c|}
\hline & & & & rity & & $\begin{array}{l}\text { renc } \\
\text { es }\end{array}$ & $\begin{array}{l}\text { abili } \\
\text { ty }\end{array}$ & $\mathbf{N}$ & & Mode \\
\hline \multirow[t]{2}{*}{5} & \multirow{2}{*}{$\begin{array}{l}\text { Drug } \\
\text { collected } \\
\text { from } \\
\text { Pharmacy } \\
\text { / send to } \\
\text { ward } \\
\text { (con't) }\end{array}$} & \multirow{2}{*}{$\begin{array}{l}\text { Drug } \\
\text { dropped } \\
\text { broken } \\
\text { missing } \\
\text { during } \\
\text { transit } \\
\text { (con't) }\end{array}$} & \multirow[t]{2}{*}{$\begin{array}{l}\text { Staff injury - } \\
\text { medical cost }\end{array}$} & 3 & $\begin{array}{l}\text { Human } \\
\text { factors i.e. } \\
\text { carelessne } \\
\text { ss }\end{array}$ & 2 & 2 & $\begin{array}{l}1 \\
2\end{array}$ & 5 & $\begin{array}{l}\text { Increase mental } \\
\text { focus }\end{array}$ \\
\hline & & & & 3 & $\begin{array}{l}\text { Environm } \\
\text { ental } \\
\text { factors i.e. } \\
\text { distraction } \\
\text { s, space, } \\
\text { lighting, } \\
\text { hindrance, } \\
\text { noise) }\end{array}$ & 2 & 2 & $\begin{array}{l}1 \\
2\end{array}$ & 5 & $\begin{array}{l}\text { Environmental } \\
\text { and workflow } \\
\text { improvement, } \\
\text { avoid hindrances }\end{array}$ \\
\hline \multirow[t]{4}{*}{6} & \multirow{4}{*}{$\begin{array}{l}\text { Double } \\
\text { check the } \\
\text { drug } \\
\text { received. }\end{array}$} & \multirow[t]{2}{*}{$\begin{array}{l}\text { Staff did not } \\
\text { double } \\
\text { check }\end{array}$} & \multirow[t]{2}{*}{$\begin{array}{l}\text { Discrepancies } \\
\text { in stock level }\end{array}$} & 2 & $\begin{array}{l}\text { In } \\
\text { adequate } \\
\text { staffing }\end{array}$ & 3 & 3 & $\begin{array}{l}1 \\
8\end{array}$ & 3 & $\begin{array}{l}\text { Reschedule } \\
\text { staffing pattern }\end{array}$ \\
\hline & & & & 2 & $\begin{array}{l}\text { Lack of } \\
\text { making } \\
\text { the check } \\
\text { a priority }\end{array}$ & 2 & 3 & $\begin{array}{l}1 \\
2\end{array}$ & 5 & $\begin{array}{l}\text { Engaging staff in } \\
\text { culture of safety } \\
\text { - importance of } \\
\text { double checking }\end{array}$ \\
\hline & & \multirow[t]{2}{*}{$\begin{array}{l}\text { Inadequate } \\
\text { checking }\end{array}$} & \multirow{2}{*}{$\begin{array}{l}\text { Potential error } \\
\text { on patient's } \\
\text { safety not } \\
\text { detected (eg } \\
\text { expiry date, } \\
\text { correct drug / } \\
\text { strength, } \\
\text { storage } \\
\text { requirement) }\end{array}$} & 4 & $\begin{array}{l}\text { Check } \\
\text { process } \\
\text { not } \\
\text { integrated } \\
\text { in } \\
\text { workflow }\end{array}$ & 3 & 3 & $\begin{array}{l}3 \\
6\end{array}$ & 1 & $\begin{array}{l}\text { Staff to } \\
\text { document } \\
\text { signature upon } \\
\text { counterchecking } \\
\text { Training on } \\
\text { correct checking }\end{array}$ \\
\hline & & & & 4 & $\begin{array}{l}\text { Heavy } \\
\text { workload }\end{array}$ & 2 & 3 & $\begin{array}{l}2 \\
4\end{array}$ & 2 & $\begin{array}{l}\text { Task assignment } \\
\text { to in-charge }\end{array}$ \\
\hline $\begin{array}{l}\mathbf{N} \\
\mathbf{o}\end{array}$ & Process & $\begin{array}{l}\text { Failure } \\
\text { Modes }\end{array}$ & Effects & $\begin{array}{l}\text { Sev } \\
\text { e- } \\
\text { rity }\end{array}$ & $\begin{array}{l}\text { Causes of } \\
\text { Failure }\end{array}$ & $\begin{array}{l}\text { Occ } \\
\text { ur- } \\
\text { renc } \\
\text { es }\end{array}$ & $\begin{array}{l}\text { Dete } \\
\text { ct- } \\
\text { abili } \\
\text { ty }\end{array}$ & $\begin{array}{l}\mathbf{R} \\
\mathbf{P} \\
\mathbf{N}\end{array}$ & $\begin{array}{l}\text { Ra } \\
\text { nk }\end{array}$ & $\begin{array}{lr}\text { Action } & \text { to } \\
\text { Reduce } & \text { Failure } \\
\text { Mode } & \end{array}$ \\
\hline \multirow[t]{6}{*}{7} & \multirow[t]{6}{*}{$\begin{array}{l}\text { Store } \\
\text { drug } \\
\text { according } \\
\text { ly in } \\
\text { ward }\end{array}$} & \multirow[t]{2}{*}{$\begin{array}{l}\text { Drug not } \\
\text { stored } \\
\text { correctly } \\
\text { (according } \\
\text { to storage } \\
\text { requirement) }\end{array}$} & \multirow[t]{2}{*}{$\begin{array}{l}\text { Damaged drug } \\
- \text { loss to } \\
\text { company }\end{array}$} & 3 & $\begin{array}{l}\text { Staff } \\
\text { unaware } \\
\text { of storage } \\
\text { condition }\end{array}$ & 2 & 3 & $\begin{array}{l}1 \\
8\end{array}$ & 3 & $\begin{array}{l}\text { Storage } \\
\text { condition } \\
\text { requirements for } \\
\text { drugs to be } \\
\text { displayed clearly } \\
\text { Training to staff }\end{array}$ \\
\hline & & & & 3 & $\begin{array}{l}\text { Staff } \\
\text { mistakenl } \\
\text { y placed } \\
\text { drug }\end{array}$ & 2 & 2 & $\begin{array}{l}1 \\
2\end{array}$ & 5 & $\begin{array}{l}\text { Bin card update } \\
5 \text { s arrangement } \\
\text { Regular check } \\
\text { on stock level }\end{array}$ \\
\hline & & $\begin{array}{l}\text { Not } \\
\text { practicing } \\
\text { FIFO }\end{array}$ & $\begin{array}{l}\text { Increased } \\
\text { expired drug - } \\
\text { loss to } \\
\text { company }\end{array}$ & 4 & $\begin{array}{l}\text { No regular } \\
\text { check }\end{array}$ & 3 & 3 & $\begin{array}{l}3 \\
6\end{array}$ & 1 & $\begin{array}{l}\text { Regular } \\
\text { checking should } \\
\text { be done } \\
\text { Highlight short } \\
\text { expiry drugs or } \\
\text { separate at } \\
\text { immediate use } \\
\text { area }\end{array}$ \\
\hline & & \multirow[t]{3}{*}{$\begin{array}{l}\text { Storage } \\
\text { space } \\
\text { inadequate }\end{array}$} & \multirow[t]{2}{*}{ 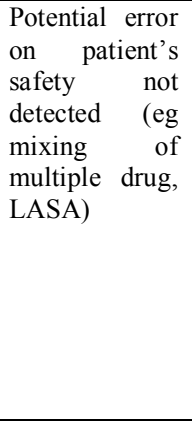 } & 4 & $\begin{array}{l}\text { Environm } \\
\text { ental } \\
\text { factors eg } \\
\text { space } \\
\text { constraint }\end{array}$ & 2 & 3 & $\begin{array}{l}2 \\
4\end{array}$ & 2 & $\begin{array}{l}\text { To allocate space } \\
\text { for drug storage } \\
5 \mathrm{~S} \text { arrangement } \\
\text { Alert tag } \\
\text { displayed, LASA } \\
\text { drug not placed } \\
\text { next to each } \\
\text { other }\end{array}$ \\
\hline & & & & 4 & $\begin{array}{l}\text { Human } \\
\text { factors eg } \\
\text { staff } \\
\text { misplace } \\
\text { drug } \\
\end{array}$ & 2 & 3 & $\begin{array}{l}2 \\
4\end{array}$ & 2 & $\begin{array}{l}\text { Drugs segregated } \\
\text { in proper } \\
\text { container and } \\
\text { clearly labelled }\end{array}$ \\
\hline & & & $\begin{array}{l}\text { Delay patient's } \\
\text { treatment }\end{array}$ & 2 & $\begin{array}{ll}\text { Lack of } \\
\text { ease on } \\
\text { finding } \\
\text { correct } \\
\text { drug }\end{array}$ & 2 & 3 & $\begin{array}{l}1 \\
2\end{array}$ & 5 & $\begin{array}{l}\text { 5S arrangement } \\
\text { Drugs segregated } \\
\text { in proper } \\
\text { container and } \\
\text { clearly labeled }\end{array}$ \\
\hline
\end{tabular}




\begin{tabular}{|c|c|c|c|c|c|c|c|c|c|c|}
\hline & & & $\begin{array}{lr}\text { Risk on drug } \\
\text { dropped } \\
\text { broken }\end{array}$ & 2 & $\begin{array}{l}\text { Cramped } \\
\text { storage } \\
\text { area }\end{array}$ & 2 & 3 & $\begin{array}{l}1 \\
2\end{array}$ & 5 & $\begin{array}{l}\text { Allocated space } \\
\text { for drug storage } \\
\text { Avoid } \\
\text { overstocking by } \\
\text { establishing par } \\
\text { level for ward } \\
\text { stock }\end{array}$ \\
\hline $\begin{array}{l}\mathbf{N} \\
\mathbf{0}\end{array}$ & Process & $\begin{array}{l}\text { Failure } \\
\text { Modes }\end{array}$ & Effects & $\begin{array}{l}\text { Sev } \\
\text { e- } \\
\text { rity }\end{array}$ & $\begin{array}{l}\text { Causes of } \\
\text { Failure }\end{array}$ & $\begin{array}{l}\text { Occ } \\
\text { ur- } \\
\text { renc } \\
\text { es }\end{array}$ & $\begin{array}{l}\text { Dete } \\
\text { ct- } \\
\text { abili } \\
\text { ty }\end{array}$ & $\begin{array}{l}\mathbf{R} \\
\mathbf{P} \\
\mathbf{N}\end{array}$ & $\begin{array}{l}\text { Ra } \\
\text { nk }\end{array}$ & $\begin{array}{lr}\text { Action } & \text { to } \\
\text { Reduce } & \text { Failure } \\
\text { Mode } & \end{array}$ \\
\hline 8 & $\begin{array}{l}\text { Segregate } \\
\text { Returned } \\
\text { Drugs }\end{array}$ & $\begin{array}{l}\text { Drug mixed } \\
\text { up } \\
\text { Disposed } \\
\text { short expiry } \\
\text { drugs which } \\
\text { can be } \\
\text { utilized }\end{array}$ & $\begin{array}{l}\text { Loss to } \\
\text { company }\end{array}$ & 3 & $\begin{array}{l}\text { Human } \\
\text { factors eg } \\
\text { staff error }\end{array}$ & 2 & 3 & $\begin{array}{l}1 \\
8\end{array}$ & 3 & $\begin{array}{l}\text { Double checking } \\
\text { with another } \\
\text { staff }\end{array}$ \\
\hline \multirow[t]{2}{*}{9} & \multirow{2}{*}{$\begin{array}{l}\text { Issue } \\
\text { short } \\
\text { expiry } \\
\text { drugs to } \\
\text { other } \\
\text { wards for } \\
\text { utilizatio } \\
n \text { or } \\
\text { return } \\
\text { back to } \\
\text { supplier }\end{array}$} & $\begin{array}{l}\text { Short expiry } \\
\text { drugs not } \\
\text { monitored - } \\
\text { expired }\end{array}$ & $\begin{array}{l}\text { ADR, allergic } \\
\text { reaction or } \\
\text { ineffective } \\
\text { treatment if } \\
\text { administered } \\
\text { to patient }\end{array}$ & 4 & $\begin{array}{l}\text { Not } \\
\text { practice } \\
\text { FIFO } \\
\text { Lack of } \\
\text { monitorin } \\
\text { g of stock } \\
\text { expiry }\end{array}$ & 2 & 3 & $\begin{array}{l}2 \\
4\end{array}$ & 2 & $\begin{array}{l}\text { Separate drugs } \\
\text { on immediate } \\
\text { use area } \\
\text { FIFO } \\
\text { arrangement } \\
\text { Regular } \\
\text { monitoring }\end{array}$ \\
\hline & & $\begin{array}{l}\text { Drugs not } \\
\text { returnable }\end{array}$ & $\begin{array}{l}\text { Loss to } \\
\text { company - } \\
\text { drugs to be } \\
\text { disposed off }\end{array}$ & 3 & $\begin{array}{l}\text { Drugs has } \\
\text { expired or } \\
\text { very short } \\
\text { expiry not } \\
\text { accepted } \\
\text { for return }\end{array}$ & 2 & 3 & $\begin{array}{l}1 \\
8\end{array}$ & 3 & $\begin{array}{l}\text { Control of drug } \\
\text { ordering for non- } \\
\text { returnable drugs }\end{array}$ \\
\hline $\begin{array}{l}1 \\
0\end{array}$ & $\begin{array}{l}\text { Expired / } \\
\text { spoilt } \\
\text { item } \\
\text { disposed }\end{array}$ & $\begin{array}{l}\text { Procedures } \\
\text { of drug } \\
\text { disposal not } \\
\text { properly } \\
\text { conducted }\end{array}$ & $\begin{array}{l}\text { Environmental } \\
\text { hazards }\end{array}$ & 4 & $\begin{array}{l}\text { Staff } \\
\text { competenc } \\
y\end{array}$ & 2 & 2 & $\begin{array}{l}1 \\
6\end{array}$ & 4 & $\begin{array}{l}\text { Training \& } \\
\text { awareness } \\
\text { SOP available in } \\
\text { work area }\end{array}$ \\
\hline
\end{tabular}

\section{STEP FOUR:}

Identify the highest Risk Priority Number (RPN) based on step three.

The Risk Priority Number (RPN) determines which processes' failure modes affects the most and used to determine the rank for us to address the failure modes with the highest RPN. From table below, it showed that failure modes that are most severe, occur often and hard to detect are ranked \# 1 as greatest hazard to our patients.

\begin{tabular}{|c|c|c|c|c|c|c|c|c|c|c|}
\hline $\begin{array}{l}\mathbf{N} \\
\mathbf{0}\end{array}$ & Process & $\begin{array}{l}\text { Failure } \\
\text { Modes }\end{array}$ & Effects & $\begin{array}{c}\text { Sev } \\
\text { e- } \\
\text { rity }\end{array}$ & $\begin{array}{l}\text { Causes of } \\
\text { Failure }\end{array}$ & $\begin{array}{l}\text { Occu } \\
\text { r- } \\
\text { renc } \\
\text { es } \\
\end{array}$ & $\begin{array}{l}\text { Dete } \\
\text { ct- } \\
\text { abilit } \\
\text { y }\end{array}$ & $\begin{array}{l}\mathbf{R} \\
\mathbf{P} \\
\mathbf{N}\end{array}$ & $\begin{array}{l}\text { Ra } \\
\text { nk }\end{array}$ & $\begin{array}{l}\text { Action } r \\
\text { Reduce } \\
\text { Mode }\end{array}$ \\
\hline 1 & $\begin{array}{l}\text { Check } \\
\text { stock } \\
\text { level in } \\
\text { ward }\end{array}$ & $\begin{array}{l}\text { SN did not } \\
\text { check ward } \\
\text { stock level. }\end{array}$ & $\begin{array}{l}\text { Patient may } \\
\text { not get } \\
\text { medication on } \\
\text { time during } \\
\text { emergency }\end{array}$ & 4 & $\begin{array}{l}\text { Replenish } \\
\text { ment } \\
\text { failure }\end{array}$ & 3 & 3 & 36 & 1 & $\begin{array}{l}\text { Integrate check } \\
\text { process into daily } \\
\text { task }\end{array}$ \\
\hline 2 & $\begin{array}{l}\text { Double } \\
\text { check the } \\
\text { drug } \\
\text { received. }\end{array}$ & $\begin{array}{l}\text { Inadequate } \\
\text { checking }\end{array}$ & $\begin{array}{l}\text { Potential error } \\
\text { on patient's } \\
\text { safety not } \\
\text { detected (eg } \\
\text { expiry date, } \\
\text { correct drug / } \\
\text { strength, } \\
\text { storage } \\
\text { requirement) }\end{array}$ & 4 & $\begin{array}{l}\text { Check } \\
\text { process } \\
\text { not } \\
\text { integrated } \\
\text { in } \\
\text { workflow }\end{array}$ & 3 & 3 & 36 & 1 & $\begin{array}{l}\text { Staff to } \\
\text { document } \\
\text { signature upon } \\
\text { counterchecking } \\
\text { Training on } \\
\text { correct checking }\end{array}$ \\
\hline 3 & $\begin{array}{l}\text { Store } \\
\text { drug } \\
\text { according } \\
\text { ly in ward }\end{array}$ & $\begin{array}{l}\text { Not } \\
\text { practicing } \\
\text { FIFO }\end{array}$ & $\begin{array}{l}\text { Increased } \\
\text { expired drug - } \\
\text { loss to } \\
\text { company }\end{array}$ & 4 & $\begin{array}{l}\text { No regular } \\
\text { check }\end{array}$ & 3 & 3 & 36 & 1 & $\begin{array}{l}\text { Regular checking } \\
\text { should be done } \\
\text { Highlight short } \\
\text { expiry drugs or } \\
\text { separate at } \\
\text { immediate use } \\
\text { area }\end{array}$ \\
\hline
\end{tabular}

In step four, it was found that check stock level in ward, double checking the drug received and store drug accordingly in ward received the highest RPN of 36. 


\section{STEP FIVE:}

One of the most important factors for the success of FMEA in any organization is an effective FMEA Process. It takes a focused strategy to bring about the infrastructure that is necessary to support effective FMEAs, but it is well worth the time and effort (Carlson, 2012). Therefore the team decided to redesign the supply process .In the new system, pharmacy staff will go to each ward to check the stock of drugs kept at the ward level. They are required to check drugs stored at ward level once a month using the following criteria:
i. $\quad$ Storage condition
ii. $\quad$ Par level (excess / shortage)
iii. Labeling \& Packaging
iv. Non-conformances (Spoilt / Expired)

The findings and corrective actions shall be documented in Ward Check Form and duly reported to the relevant personnel (Chief Pharmacist and Unit Managers). Findings for each ward are scored accordingly and tabulated.

In June 2011, a new process of indenting of drugs for ward stock was implemented.

\section{New Process Of Monthly Ward Stock Check By Pharmacy Staff}

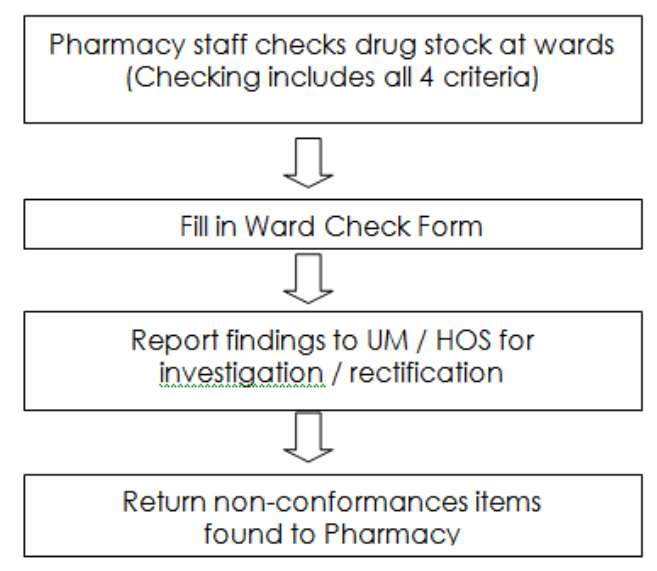

STEP SIXTH:

After implementing the new process, another FMEA was done.

V. Fmea On New Process Of Monthly Ward Check By Pharmacy Staff:

\begin{tabular}{|c|c|c|c|c|c|c|c|c|c|c|}
\hline $\begin{array}{l}\mathbf{N} \\
\mathbf{0}\end{array}$ & Process & $\begin{array}{l}\text { Failure } \\
\text { Modes }\end{array}$ & Effects & $\begin{array}{l}\text { Sev } \\
\text { e- } \\
\text { rity }\end{array}$ & $\begin{array}{l}\text { Causes of } \\
\text { Failure }\end{array}$ & $\begin{array}{l}\text { Occ } \\
\text { ur- } \\
\text { renc } \\
\text { es }\end{array}$ & $\begin{array}{l}\text { Dete } \\
\text { ct- } \\
\text { abili } \\
\text { ty }\end{array}$ & $\begin{array}{l}\mathbf{R} \\
\mathbf{P} \\
\mathbf{N}\end{array}$ & $\begin{array}{l}\text { Ra } \\
\text { nk }\end{array}$ & $\begin{array}{lr}\text { Action } & \text { to } \\
\text { Reduce } & \text { Failure } \\
\text { Mode } & \end{array}$ \\
\hline \multirow[t]{4}{*}{1} & \multirow{4}{*}{$\begin{array}{l}\text { Pharmacy } \\
\text { staff } \\
\text { checks } \\
\text { drug } \\
\text { stock at } \\
\text { wards } \\
\text { (Checkin } \\
\text { g includes } \\
\text { all } 4 \\
\text { criteria) }\end{array}$} & \multirow[t]{2}{*}{$\begin{array}{l}\text { Staff did not } \\
\text { carry out } \\
\text { ward check }\end{array}$} & \multirow[t]{2}{*}{$\begin{array}{l}\text { Ward stock is } \\
\text { not properly } \\
\text { stored and } \\
\text { overstock }\end{array}$} & 3 & $\begin{array}{l}\text { Human } \\
\text { factors e.g } \\
\text { staff } \\
\text { forgot }\end{array}$ & 2 & 1 & 6 & 2 & $\begin{array}{lr}\begin{array}{l}\text { Monitoring } \\
\text { octivity }\end{array} \\
\text { Pharmacist } \\
\text { Impose as } \\
\text { KPI }\end{array}$ \\
\hline & & & & 3 & $\begin{array}{l}\text { Lack of } \\
\text { making } \\
\text { the check } \\
\text { a priority }\end{array}$ & 2 & 1 & 6 & 2 & $\begin{array}{l}\text { Engaging staff in } \\
\text { culture of safety } \\
\text { Training \& } \\
\text { awareness }\end{array}$ \\
\hline & & \multirow[t]{2}{*}{$\begin{array}{l}\text { Inadequate } \\
\text { checking }\end{array}$} & \multirow{2}{*}{$\begin{array}{l}\text { Potential error } \\
\text { on patient's } \\
\text { safety not } \\
\text { detected (eg } \\
\text { expiry date, } \\
\text { correct drug / } \\
\text { strength, } \\
\text { storage } \\
\text { requirement) }\end{array}$} & 4 & $\begin{array}{l}\text { Staff do } \\
\text { not } \\
\text { understand } \\
\text { how to } \\
\text { check }\end{array}$ & 2 & 1 & 8 & 1 & $\begin{array}{l}\text { Training \& } \\
\text { Briefing } \\
\text { Reinforce in } \\
\text { services meeting } \\
\text { Monitoring by } \\
\text { the Pharmacist }\end{array}$ \\
\hline & & & & 4 & $\begin{array}{l}\text { Staff } \\
\text { competenc } \\
y-\text { new } \\
\text { staff }\end{array}$ & 2 & 1 & 8 & 1 & Training \\
\hline 1 & $\begin{array}{l}\text { (con't..) } \\
\text { Pharmacy } \\
\text { staff } \\
\text { checks }\end{array}$ & $\begin{array}{l}\text { Inadequate } \\
\text { checking }\end{array}$ & 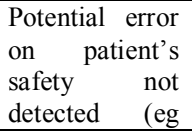 & 4 & $\begin{array}{l}\text { Not using } \\
\text { ward } \\
\text { check } \\
\text { form as }\end{array}$ & 2 & 1 & 8 & 1 & $\begin{array}{l}\text { Make sure form } \\
\text { is available all } \\
\text { the times }\end{array}$ \\
\hline
\end{tabular}




\begin{tabular}{|c|c|c|c|c|c|c|c|c|c|c|}
\hline & $\begin{array}{l}\text { drug } \\
\text { stock at } \\
\text { wards } \\
\text { (Checkin } \\
\text { g includes } \\
\text { all } 4 \\
\text { criteria) }\end{array}$ & & $\begin{array}{l}\text { expiry date, } \\
\text { correct drug / } \\
\text { strength, } \\
\text { storage } \\
\text { requirement) }\end{array}$ & & references & & & & & \\
\hline \multirow[t]{2}{*}{2} & \multirow[t]{2}{*}{$\begin{array}{l}\text { Fill in } \\
\text { Ward } \\
\text { Check } \\
\text { Form }\end{array}$} & $\begin{array}{l}\text { Form } \\
\text { incomplete }\end{array}$ & $\begin{array}{l}\text { Pharmacist / } \\
\text { UM is not } \\
\text { informed on } \\
\text { any non- } \\
\text { conformances }\end{array}$ & 3 & $\begin{array}{l}\text { Staff do } \\
\text { not } \\
\text { understand } \\
\text { how to fill } \\
\text { in the } \\
\text { form }\end{array}$ & 2 & 1 & 6 & 2 & $\begin{array}{l}\text { Training \& } \\
\text { Briefing } \\
\text { Reinforce in } \\
\text { services meeting } \\
\begin{array}{l}\text { Form to be } \\
\text { verified } \\
\text { Pharmacist }\end{array}\end{array}$ \\
\hline & & $\begin{array}{l}\text { Form is not } \\
\text { filled in }\end{array}$ & $\begin{array}{l}\text { No } \\
\text { documentation }\end{array}$ & 3 & $\begin{array}{l}\text { Form not } \\
\text { available }\end{array}$ & 2 & 1 & 6 & 2 & $\begin{array}{l}\text { Make sure form } \\
\text { is available all } \\
\text { the times } \\
\text { routine } \\
\text { monitoring by } \\
\text { Pharmacy Clerk }\end{array}$ \\
\hline 3 & $\begin{array}{l}\text { Report } \\
\text { findings } \\
\text { to UM / } \\
\text { HOS for } \\
\text { investigat } \\
\text { ion } \\
\text { /rectificat } \\
\text { ion }\end{array}$ & \begin{tabular}{ll}
\multicolumn{2}{l}{ Findings } \\
was not \\
reported
\end{tabular} & $\begin{array}{l}\text { Discrepancies } \\
\text { was not } \\
\text { rectified - risk } \\
\text { of potential } \\
\text { error to patient } \\
\text { safety is not } \\
\text { minimize }\end{array}$ & 4 & $\begin{array}{l}\text { Human } \\
\text { factors e.g } \\
\text { staff } \\
\text { forgot, } \\
\text { staff } \\
\text { competenc } \\
\text { y }\end{array}$ & 2 & 1 & 8 & 1 & $\begin{array}{ll}\text { Reinforce } & \\
\text { verification } & \text { by } \\
\text { UM } & \& \\
\text { Pharmacist } & \end{array}$ \\
\hline \multirow[t]{3}{*}{4} & \multirow{3}{*}{$\begin{array}{l}\text { Return } \\
\text { non- } \\
\text { conforma } \\
\text { nces } \\
\text { items } \\
\text { found to } \\
\text { Pharmacy }\end{array}$} & \multirow[t]{3}{*}{$\begin{array}{l}\text { Drug } \\
\text { dropped } \\
\text { broken } \\
\text { during } \\
\text { transit }\end{array}$} & $\begin{array}{ll}\text { Cost } & \text { to } \\
\text { company }- \text { to } \\
\text { replace loss of } \\
\text { drug }\end{array}$ & 3 & $\begin{array}{l}\text { Security of } \\
\text { drugs } \\
\text { during } \\
\text { transit }\end{array}$ & 1 & 1 & 3 & 3 & $\begin{array}{l}\text { Medication box } \\
\text { use to transfer } \\
\text { drugs }\end{array}$ \\
\hline & & & \multirow[t]{2}{*}{$\begin{array}{l}\text { Staff injury - } \\
\text { medical cost }\end{array}$} & 3 & $\begin{array}{l}\text { Human } \\
\text { factors i.e. } \\
\text { carelessne } \\
\text { ss }\end{array}$ & 1 & 1 & 3 & 3 & $\begin{array}{l}\text { Increase mental } \\
\text { focus }\end{array}$ \\
\hline & & & & 3 & $\begin{array}{l}\text { Environm } \\
\text { ental } \\
\text { factors i.e. } \\
\text { distraction } \\
\text { s, space, } \\
\text { lighting, } \\
\text { hindrance, } \\
\text { noise) }\end{array}$ & 1 & 1 & 3 & 3 & $\begin{array}{l}\text { Environmental } \\
\text { and workflow } \\
\text { improvement, } \\
\text { avoid hindrances }\end{array}$ \\
\hline
\end{tabular}

Based on the new FMEA it was found that although the severity score is high, the occurrences level and detectability score is low as the activity is conducted monthly under the Pharmacist's supervision and reported to unit managers. Therefore, RPN is also low which indicates improvement in terms of minimizing the risk of hazardous effects .

\section{STEP SEVEN:}

To monitor the new process using ward check form based on the following criteria:

i) Storage condition

ii) Par level (excess / shortage)

iii) Labelling \& Packaging

iv) Non-conformances (Spoilt / Expired)

Scoring mechanism is as follows:

1 - No discrepancies observed

0 - Discrepancies observed such as very short expiry /expired drugs, exceeded par level, no label, incorrect storage condition. 


\section{Results}

1. Data of drug disposed

\begin{tabular}{|l|l|l|l|}
\hline & $\begin{array}{l}\text { January-December 2010 } \\
\text { (old system for drug ordering) }\end{array}$ & $\begin{array}{l}\text { July 2011 -June 2012 } \\
\text { (new system for drug ordering) }\end{array}$ & Variance \\
\hline Value of drugs disposed & RM 8,575.50 & RM 3,060.78 & $94 \%$ \\
\hline
\end{tabular}

2. Checking compliance for drug storage

Checking of compliance at ward level was implemented in July 2011.

Percentage (\%) of Compliance on Overall Drug Storage in Wards

\begin{tabular}{|l|l|l|l|l|l|l|l|l|l|l|l|l|l|}
\hline CRITERIA & $\begin{array}{l}\text { JULY } \\
\mathbf{2 0 1 1}\end{array}$ & $\begin{array}{l}\text { AUG } \\
\mathbf{2 0 1 1}\end{array}$ & $\begin{array}{l}\text { SEPT } \\
\mathbf{2 0 1 1}\end{array}$ & $\begin{array}{l}\text { OCT } \\
\mathbf{2 0 1 1}\end{array}$ & $\begin{array}{l}\text { NOV } \\
\mathbf{2 0 1 1}\end{array}$ & $\begin{array}{l}\text { DEC } \\
\mathbf{2 0 1 1}\end{array}$ & $\begin{array}{l}\text { JAN } \\
\mathbf{2 0 1 2}\end{array}$ & $\begin{array}{l}\text { FEB } \\
\mathbf{2 0 1 2}\end{array}$ & $\begin{array}{l}\text { MAC } \\
\mathbf{2 0 1 2}\end{array}$ & $\begin{array}{l}\text { APR } \\
\mathbf{2 0 1 2}\end{array}$ & $\begin{array}{l}\text { MAY } \\
\mathbf{2 0 1 2}\end{array}$ & $\begin{array}{l}\text { JUNE } \\
\mathbf{2 0 1 2}\end{array}$ & $\begin{array}{l}\text { TOTAL } \\
\text { STORAGE } \\
\text { CONDITION }\end{array}$ \\
100 & 100 & 100 & 100 & 100 & 100 & 100 & 100 & 100 & 100 & 100 & 100 & 100 \\
\hline PAR LEVEL & 91.7 & 91.7 & 83.3 & 50 & 66.7 & 66.7 & 91.7 & 75 & 91.7 & 91.7 & 92.0 & 91.7 & 81.9 \\
\hline LABELLING & 91.7 & 100 & 100 & 100 & 100 & 100 & 100 & 91.7 & 91.7 & 91.7 & 100 & 100 & 97.2 \\
\hline $\begin{array}{l}\text { EXPIRY } \\
\text { DATE } \\
\text { STILL } \\
\text { VALID }\end{array}$ & & & & & & & & & & & & \\
\end{tabular}

\section{Discussion}

Before implementing the FMEA, the value of drugs disposed was RM 8,775.50 due to expired or spoiled items returned from wards. After implementing the new system based on the FMEA the value of drugs disposed reduced to RM 3,060.78. Therefore there was $94 \%$ reduction in term of drug wastage. The main reason is because in the old system, checking of drugs at ward level was done by nurses when they plan to indent the new stock. In the new system, checking are done by pharmacy staff regularly once a month. Monthly report will be given to all unit managers . Compliance in term of drug storage was $100 \%$ and labeling compliance was $97.2 \%$. However the compliance on par level was only $81.9 \%$. Therefore drugs exceeding par level will be taken back to pharmacy department. In term of expiry date validity, the compliance was $96.5 \%$. Based on the FMEA tool, it was found that the main reason for expired /spoiled drugs is because of insufficient checking of ward stocks. Corrective actions are ongoing to improve further the process of supply by taking the following actions:

- Re-designing a new form of ward checking to ensure all drugs storage areas at wards are checked

- Re-modifying indenting process of ward stocks via the HITS system, where wards will be ordering drugs through HITS and Pharmacy can monitor ward stock level before replenishment to the respective wards.

- Conducting training \& awareness on the importance of drug safety and its risk for new staff

\section{Conclusion}

FMEA was found to be an effective tool for identifying potential problems and the same tool can be applied to other process in the hospital. The study has shown remarkable results in reducing amount of drug disposal in KPJ Seremban which will help the organization to reduce the risk to patients and avoid wastage.

\section{References}

[1]. National Centre for Patient Safety, Healthcare Failure Mode and Effect Analysis (HFMEA), Institute for Safe Medication Practic es (ISMP) 2005

[2]. JCI, Failure Mode and Effects Analysis in Health Care: Proactive Risk Reduction, Third Edition ,p 20, 2010

[3]. McDermott R.E et.al, The Basic FMEA, New York: Productivity Press, 2009,p.17

[4]. Tischler L : Seven secrets to good brain storming. http:// www.fastcompany.com/articles/2001/03/kelley.html,Mar. 4, 2010

[5]. Crow K, Failure mode and effect analysis (FMEA), 2002.

[6]. InstituteofHealthImprovement,2013:http://www.ihi.org/knowledge/Pages/Measures/RiskPriorityNumberfromFailureModesandEffe ctsAnalysis.aspx

[7]. Stamatis, D.H., Failure Mode and Effect Analysis: FMEA from Theory to Execution. American Society for Quality (ASQ), Milwaukee, Wisconsin, 1995.

[8]. Carl S.Carlson, FMEA success factors: an effective FMEA process, Reliability edge home, volume 6, Issue 1,2009

[9]. Wetterneck TB, Hundt AS,Carayon P, FMEA team performance in health care: A qualitative analysis of a team member perceptions, J Patient Saf . 2009 June; 5(2): 102-8

[10]. Fareza, Pharmacy Services report, ed.8, 2010, p .20-25

[11]. Fareza, Pharmacy Services report, ed. 9, 2011, p . 30-35

[12]. Fareza, Pharmacy Services report, ed. 10, 2012, p. 30-35 